\title{
Profusion of transition pathways for interacting hysterons
}

\author{
Martin van Hecke \\ AMOLF, Science Park 104, 1098 XG Amsterdam, Netherlands \\ and Huygens-Kamerlingh Onnes Lab, Universiteit Leiden, P.O. Box 9504, NL-2300 RA Leiden, Netherlands
}

(Received 14 July 2021; accepted 14 October 2021; published 12 November 2021)

\begin{abstract}
The response, pathways, and memory effects of cyclically driven complex media can be captured by hysteretic elements called hysterons. Here we demonstrate the profound impact of hysteron interactions on pathways and memory. Specifically, while the Preisach model of independent hysterons features a restricted class of pathways which always satisfy return point memory, we show that three interacting hysterons generate more than 15000 transition graphs, with most violating return point memory and having features completely distinct from the Preisach model. Exploring these opens a route to designer pathways and information processing in complex matter.
\end{abstract}

DOI: 10.1103/PhysRevE.104.054608

\section{INTRODUCTION}

A rugged landscape governs the intermittent response of complex materials, which features sequences of transitions between metastable states that constitute pathways [1-18]. Under cyclical driving, these pathways form hysteresis loops which may encode return point memory (RPM), the widespread ability of complex materials to revisit a previous state when the driving reaches a previous extremal value [15-21]. Microscopically, localized bistable entities such as spins or rearranging zones play a crucial role $[13,15,19-23]$. These can be modeled as hysterons, twostate elements which switch between phases " 0 " and " 1 " when the global driving field $U$ passes through the upper and lower "bare" switching fields $u_{i}^{+}$or $u_{i}^{-}$(Fig. 1) [13,15-21,24-27]. Indeed, collections of noninteracting hysterons-the well-studied Preisach model-describe surprisingly complex sequences of transitions and satisfy RMP [13,16,17,20,21,27].

Here we probe the rich physics of interacting hysterons. Interactions are physically expected $[13,17,22,25,26]$ and while noninteracting hysterons switch their phases indepedently of each other, in sequences determined by the order of their bare switching fields, interactions scramble and entangle these switching orders. To characterize the response of coupled hysterons we use transition graphs (t-graphs), a recently introduced representation that captures all pathways of a complex system and aids in probing memory effects [13,20-27].

We investigate t-graphs of $n$ interacting hysterons, focusing on $n=2$ and $n=3$. We show that interactions mushroom the number of t-graphs, which feature transitions (avalanches, pseudoavalanches, multiedges) and topological structures (subharmonic cycles, breakdown of RPM) completely distinct from the Preisach model. Besides providing a fresh perspective on the response of complex media, we show that our study paves the way for materials with designer pathways [27-31].

\section{MODEL AND T-GRAPH CONSTRUCTION}

In this section we describe the model of linearly interacting hysterons in detail. We first describe the models elementary state transitions (Sec. II A). We then introduce a recursive algorithm to obtain t-graphs for arbitrary realizations of the model (Sec. II B), and for reference briefly show the t-graphs for the Preisach model (Sec. II C).

\section{A. Model}

We model interactions via a linear dependence of the switching fields $U_{i}^{ \pm}$of hysteron $i$ on the phases $s_{j}$ of all other hysterons (Fig. 1):

$$
U_{i}^{+,-}(S)=u_{i}^{+,-}-\Sigma_{j \neq i} c_{i j} s_{j},
$$

where $S$ denotes the state $\left\{s_{1}, s_{2}, \ldots\right\}$ and $u_{i}^{+,-}$and $c_{i j}$ are the bare switching fields and interactions constants $[25,26]$. We take $0<u_{i}^{-}<u_{i}^{+}<1$, require $u_{1}^{+}>u_{2}^{+}>\ldots$ and assume no degeneracies to occur [20,21]. Furthermore, we choose a gauge where $c_{i i}=0$; note that nonzero diagonal interaction constants $c_{i i}$ only affect the values of $U_{i}^{-}$and can readily be absorbed in the bare switching fields $u_{i}^{-}$.

The upper and lower switching fields for a given state $S$ follow from the minimum (maximum) value of the switching fields of each hysteron:

$$
\begin{aligned}
& U^{+}(S)=\min _{i_{0}} U_{i_{0}}^{+}(S), \\
& U^{-}(S)=\max _{i_{i}} U_{i_{1}}^{-}(S),
\end{aligned}
$$

where $i_{0}\left(i_{1}\right)$ runs over the hysterons that are $0(1)$. State $S$ becomes unstable when $U$ exceeds $U^{+}(S)$ or decreases below $U^{-}(S)$, which initiates an up or down transition at critical driving value $U^{c}=U^{ \pm}(S)$ (note that extremal states have only one transition).

Interactions can induce avalanches that proceed via intermediate, unstable states. For example, consider the case where 

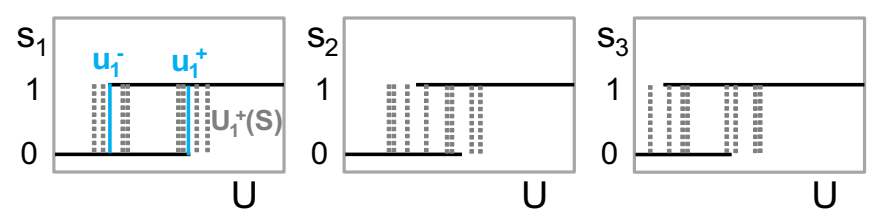

FIG. 1. Hysteresis diagrams for three interacting hysterons driven by a global field $U$. A single hysteron features internal phase $s_{i}=0,1$ and bare switching fields $u_{i}^{+,-}$(blue, full line). Interactions lead to multiple state-dependent switching fields $U_{i}^{+,-}(S)$ (gray, dashed) for each hysteron.

$S$ initially transitions to a state $S^{\prime}$ at value of $U$ where $S^{\prime}$ is not stable, and then transitions to a stable landing state $S^{\prime \prime}$. We call this an avalanche of length two, denote it as $S \rightarrow S^{\prime \prime}$, and ignore the intermediate states which in experiments would not be observable. By considering the stability of all hysterons in the transition state $S^{\prime}$ at $U=U^{c}$, the following scenario's can arise (Fig. 2).

(i) The transition state $S^{\prime}$ is a stable landing state when $U^{-}\left(S^{\prime}\right)<U^{c}<U^{+}\left(S^{\prime}\right)$ yielding a transition between state $S$ and $S^{\prime}$ [Fig. 2(a)]. While $S^{\prime}$ is always stable at $U=U^{c}$ when $c_{i j}=0$, interactions may cause state $S^{\prime}$ to be unstable, leading to avalanches.

(ii) When one " 0 " hysteron of $S^{\prime}$ is unstable, there is an additional up transition from $S^{\prime}$ to $S^{\prime \prime}$ [Fig. 2(b)], while when one " 1 " hysteron is unstable, there is an additional down transition from $S^{\prime}$ to $S^{\prime \prime}$ [Fig. 2(c)]. If state $S^{\prime \prime}$ is stable, then we obtain an avalanche of length two between state $S$ and $S^{\prime \prime}$ in which two hysterons change their phase at fixed $U=U^{c}$; if state $S^{\prime \prime}$ is unstable, we iterate and may find longer avalanches.

(iii) Occasionally more than one hysteron may be unstable in a given state [Fig. 2(d)]. Despite not having been discussed before to the best of our knowledge, such scenario can readily be constructed. In fact, we have observed that such conditions can arise for arbitrarily weak interactions, and have a significant statistical weight for stronger interactions (see Secs. III and IV). Hence, they are not a mathematical subtlety, but are an intrinsic feature of arbitrarily linearly coupled hysterons. We note that extensions of the model could be considered

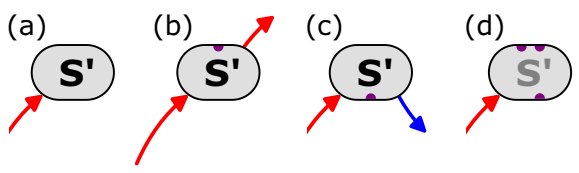

FIG. 2. Four scenario's after an up-transition reaches the state $S^{\prime}$ at $U=U^{c}$ (red incoming arrow coming from below), depending on the stability of each hysteron in state $S^{\prime}$ at $U=U^{c}$; unstable hysterons in the 0 (1) phase are visualized as (purple) dots at the top (bottom) of the node $S^{\prime}$. (a) The transition terminates at $S^{\prime}$ when all hysterons are stable at $U=U^{c}$. (b) If one phase 0 hysteron is unstable at $U=U^{c}$, then this hysteron switches to phase 1 , defining an additional step of the transition that now forms an avalanche (outgoing arrow, red). (c) If one phase 1 hysteron is unstable at $U=U^{c}$, then this hysteron switches to phase 1 , defining an additional step of the transition that now forms an avalanche (outgoing arrow, blue). (d) If more than one hysteron is unstable at $U=U^{c}$, then we consider the model ill-defined. so that the "most unstable" hysteron would switch and define state $S^{\prime \prime}$. However, here we choose to consider the corresponding t-graph to be ill-defined whenever a state with multiple unstable hysterons arises.

\section{B. Recursive algorithm and transition graphs}

We collect all transitions between states that can be reached from the extremal states $\mathbf{0}=\{0,0, \ldots\}$ or $\mathbf{1}=\{1,1, \ldots\}$ into a t-graph via an iterative algorithm. Starting from state $\{\mathbf{0}\}$, we determine its up transition and landing state, and then iteratively determine the transitions from all fresh landing states, until no new fresh states can be found. The resulting t-graph contains all $N$ nodes that are reachable from the extremal states and $2 N-2$ directed edges which represent the transitions, where each edge is labeled by its character (up or down), its critical field $U^{+}$or $U^{-}$, and the number of intermediate steps.

We collect transitions between states that can be reached from the ground state $\mathbf{0}=\{0,0, \ldots\}$ into a t-graph via an iterative algorithm. This algorithm initiates a t-graph by node $\mathbf{0}$ and determines its up transition to a state $S_{1}$ following the procedure outlined above. Node $S_{1}$ is then added to the graph, as well as the edge $\mathbf{0} \rightarrow S_{1}$. Node $\mathbf{0}$ is than labeled as "stale," as all its transitions have been determined, and $S_{1}$ is labeled as a "fresh" node. The up (provided $S_{1} \neq \mathbf{1}=\{1,1, \ldots\}$ ) and down transitions from the fresh state $S_{1}$ to states $S_{2}$ and $S_{3}$ are determined, state $S_{1}$ is labeled as stale, and states $S_{2}$ and $S_{3}$ are added to the t-graph and labeled as fresh provided they have not been visited before. This procedure is repeated until no more new fresh states are found.

Occasionally, loop-like avalanches of the form $S \rightarrow$ $S^{\prime} \cdots \rightarrow S$ occur: we consider the corresponding t-graphs ill-defined. Again, we note that such cases can readily be constructed (we give an explicit example in Sec. IIIB) and carry a significant weight. We suggest that more elaborate models, which for example would have an energy functional and dissipation, could be constructed to avoid such self-loops; for simple linearly coupled hysterons, they are an intrinsic feature of the model.

The resulting t-graph contains all $N$ nodes that are connected to state $\mathbf{0}$ and the $2 N-2$ directed edges which represents the transitions, where each edge is labeled by its character (up or down transition), its corresponding critical field $U^{c}$, and its length, i.e., the number of steps in an avalanche (one for an ordinary transition). Graphically, we order the nodes from bottom to top as function of their magnetization $m=\Sigma s_{i}$, and from left to right lexicographically. Up and down transitions are colored red and blue, and the thickness of the edges represents the length of the avalanche.

T-graph construction involves evaluating "design" inequalities on the parameters which govern each transition. These inequalities are not independent and vary strongly with the t-graph topology. Moreover, certain parameters, in particular for strong interactions, may yield avalanches that return to their initial state (self-loops), or contain states where more than one hysteron is unstable; we consider the corresponding t-graphs ill-defined. Together, this makes finding and classifying all t-graphs complex. 

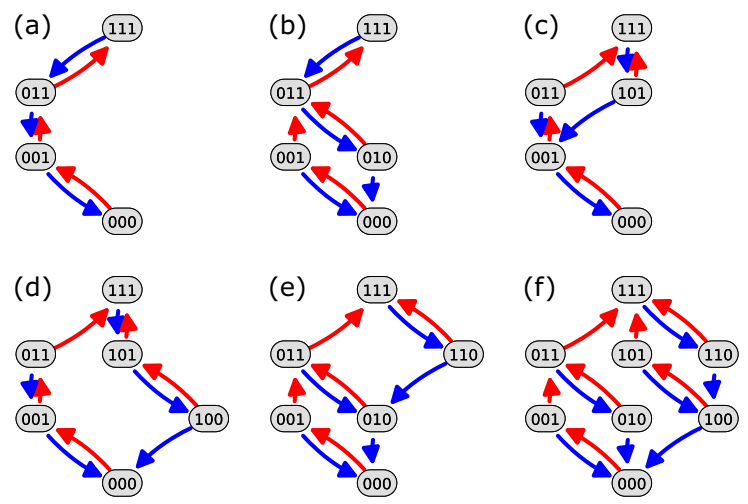

FIG. 3. T-graphs for the $n=3$ Preisach model (no interactions), for $u_{1}^{+}>u_{2}^{+}>u_{3}^{+}$. Up and down transitions are represented by red (light gray) and blue (dark gray) arrows, respectively. The ordering of the lower switching fields determines each graph [20,21]: (a) $u_{1}^{-}>$ $u_{2}^{-}>u_{3}^{-}$. (b) $u_{1}^{-}>u_{3}^{-}>u_{2}^{-}$. (c) $u_{2}^{-}>u_{1}^{-}>u_{3}^{-}$. (d) $u_{2}^{-}>u_{3}^{-}>u_{1}^{-}$. (e) $u_{3}^{-}>u_{1}^{-}>u_{2}^{-}$. (f) $u_{3}^{-}>u_{2}^{-}>u_{1}^{-}$.

\section{Physical relevance}

The model that we discuss here is perhaps the simplest model in which hysterons are interacting, and as such is a natural choice, also studied in Refs. $[25,26]$. One might wonder if in physical systems, the interaction coefficients are free or are subject to additional constraints, such as reciprocity. While more work is needed, experiments on crumpled sheets in which such interactions can be measured do not find any evidence for such constraints [13,32]. In fact, these experiments suggest that the coupling coefficient $c_{i j}$ may take on different values-including with different signs-for the upper and lower switching field, so that twice as much interactions coefficients might be needed to describe the full experiments [13]. Nevertheless, the current model, despite having less free parameters, contains t-graphs of similar complexity as found experimentally. Finally, we stress that bistable elements are a work horse of mechanical metamaterials, and we are already finding that coupling these appropriately yields experimental realizations of interacting hysterons with accompanying complex t-graphs [33]. Hence, while much more work is needed, the model studied here provides a solid jumping point for both experimental and more advanced theoretical studies.

\section{Preisach t-graphs}

In the absence of interactions, the model is exactly the Preisach model, whose t-graphs and properties have recently received renewed attention [20,21]. Under the assumed ordering of the upper switching fields, each permutation of the ordering of the lower switching fields yields a unique t-graph, that we include here (for $n=3$ ) to gain familiarity with our representation of the t-graphs and for comparison (Fig. 3). We note that the two $n=2$ Preisach t-graphs are equivalent to the subgraphs of Fig. 2 obtained by pruning the $\{1 x x\}$ states.

\section{TWO INTERACTING HYSTERONS}

In this section, we determine all possible t-graphs for two interacting hysterons by exhaustively sampling the parameter space span by the four bare switching fields $u_{i}^{+,-}$and two
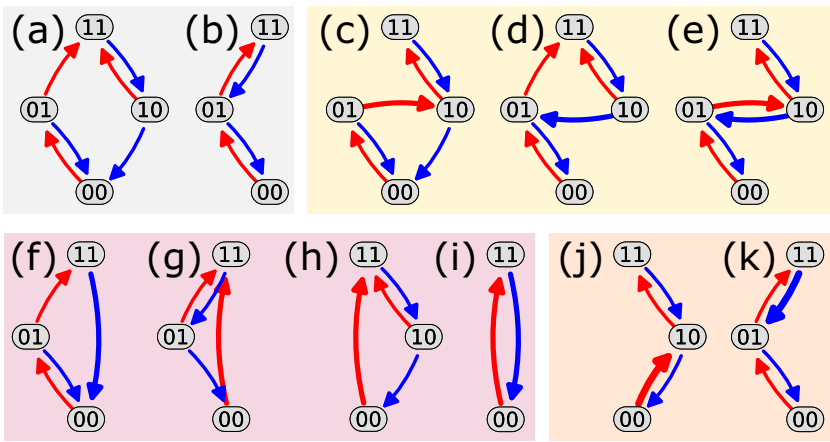

FIG. 4. Distinct transition graphs for $n=2$ coupled hysterons. (a, b) For $c_{i j}=0$ we recover the well-known Preisach t-graphs [20,21]. (c-e) For $c_{i j}<0$, "horizontal" avalanches of length two (thick arrows) may occur. (f-i) For $c_{i j}>0$, four additional t-graphs with vertical avalanches may occur. (j, k) For $c_{i j}$ of mixed sign, two additional t-graphs featuring pseudoavalanches of length three (thick arrows) are observed.

coupling coefficients $c_{12}$ and $c_{21}$ (Sec. III A). We then work out the precise constraints on these parameters for each $t$ graph to occur (Sec. III B). Finally, we present their statistical likelihood as function of interaction strength and uncover powerlaw scaling (Sec. III C).

Our results show that the underlying inequalities on the design parameters already become numerous and have an intricate structure for $n=2$, reflecting the complexity of the underlying problem. Crucially, our results clarify and quantify how even weak interactions lead to a significant growth in the number and variety of t-graphs.

\section{A. Novel t-graphs due to interactions}

Without interactions, there are only two distinct t-graphs [Figs. 4(a) and 4(b)], selected by the sign of $u_{1}^{-}-u_{2}^{-}[20,21]$. For antiferromagnetic interactions, where $c_{i j} \leqslant 0$ and the flipping of hysteron $j$ from $0 \rightarrow 1$ suppresses the flipping of hysteron $i$ from $0 \rightarrow 1$, we obtain three additional $\mathrm{t}$ graphs which feature avalanches $(\{01\} \rightarrow\{10\})$ of length two [Figs. 4(c)-(e)], while for purely ferromagnetic interactions $\left(c_{i j} \geqslant 0\right)$, there are four additional t-graphs featuring avalanches $\{00\} \rightarrow\{11\}$ [Figs. 4(f)-(i)]. For interactions of mixed sign, two additional t-graphs that both feature pseudoavalanches of length three occur [Figs. 4(j) and 4(k)] - e.g., the transition $\{00\} \rightarrow\{10\}$ in t-graph (j) proceeds via intermediate states $\{01\}$ and $\{11\}$. We note that for strong coupling, approximately $6 \%$ of parameters yield ill-defined t-graphs (see Sec. II A). We conclude that for two hysterons, interactions yield qualitatively new transitions-avalanches and pseudoavalanches - and signficantly increase the number and diversity of t-graphs.

\section{B. Design inequalities for $\boldsymbol{n}=\mathbf{2}$ interacting hysterons}

One advantage of studying the case of only two interacting hysterons is that this allows to summarize the full set of conditions on the design parameters $u_{i}^{ \pm}, c_{12}$ and $c_{21}$ for each of the $11 \mathrm{t}$-graphs of $n=2$ interacting hysterons. These inequalities give insight into the nature of the design problem 


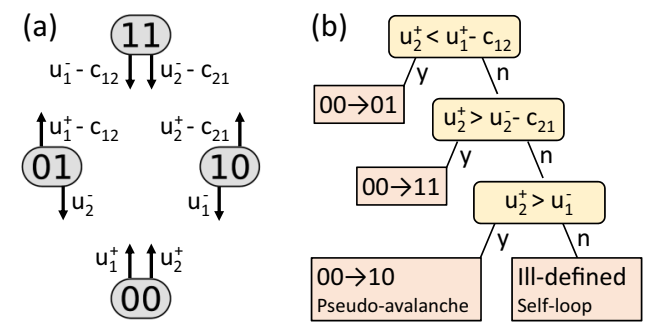

FIG. 5. (a) Switching fields of each hysteron in each potential state of two interacting hysterons. Up and won arrows indicate up and down transitions; left and right positioning indicates switching fields for hysteron 1 and 2, respectively. (b) Decision tree summarizing the conditions for transitions starting from state $\{00\}$.

and the statistics as discussed in Sec. IIIC. Unfortunately, obtaining these inequalities is slightly tedious; after providing the general idea we focus on a single subcase to guide the reader (Fig. 5), before stating the final results in Table I.

We recall the following design constraints on the bare switching fields:

$$
\begin{aligned}
& u_{2}^{+}<u_{1}^{+}, \\
& u_{1}^{-}<u_{1}^{+}, \\
& u_{2}^{-}<u_{2}^{+} .
\end{aligned}
$$

To systematically determine the conditions for all potential $\left(2 \times 2^{n}-2\right)$ up and down transitions, we first determine the $n \cdot 2^{n}$ switching fields of each hysteron [Fig. 5(a)]. We then

TABLE I. Top: Nine conditions $x_{i}$ on the design parameters. Bottom: Necessary and sufficient conditions for each $n=2 \mathrm{t}$-graph $(\mathrm{a}-\mathrm{k})$ as well as for ill-defined t-graphs.

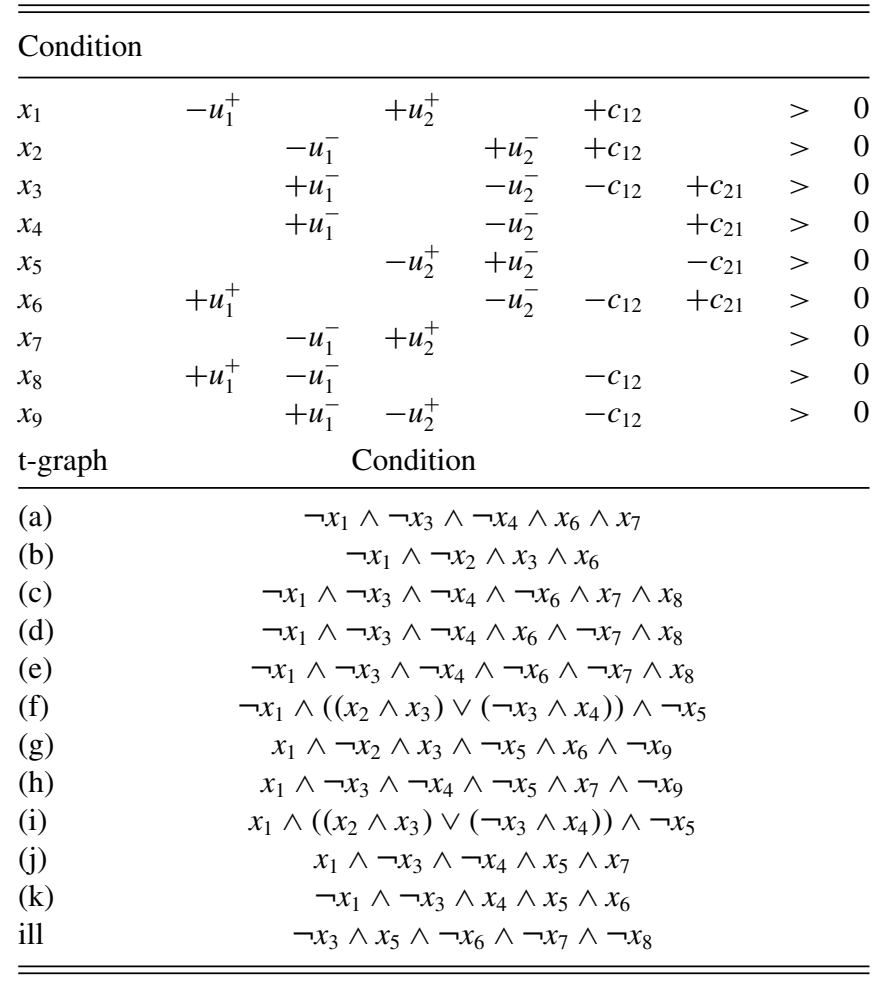

choose an initial state $S$ and proceed via a three step process: (i) We determine the conditions for the switching hysteron and the concommitant switching field $U^{c}(S)$. (We note that while for $n=2$ only the down transition from state $\{11\}$ yields a nontrivial condition, for larger $n$ the situation is more elaborate.) (ii) We consider the stability conditions of the transition state $S^{\prime}$ at $U^{c}$. If $S^{\prime}$ is stable, then it is a landing state, and we have found an $S \rightarrow S^{\prime}$ transition. (iii) When one stability condition for $S^{\prime}$ is not met it is unstable, an avalanche occurs with a new switching hysteron and towards the next transition state $S^{\prime}$ - the critical U remains as determined in the first step. For this new state, we repeat steps (ii)-(iii). When more than one stability condition is not met, the avalanche is ill-defined. Similarly, when $S^{\prime}=S$, the system is ill-defined as this causes a loop.

Starting out at state $S$, these three steps yield a set of inequalities for all transitions starting out at $S$ which span a decision tree. We note that the number of inequalities quickly grows with the number of steps in an avalanche (i.e., deeper into the three) and also with the number of hysterons, consistent with the explosive growth in the variety of t-graphs with $n$. Repeating this exercise for all states $S$, and collecting the inequalities in all corresponding threes, while tedious, yields a complete set of necessary and sufficient conditions on the design parameters for each t-graph, as well as conditions for the t-graph to be ill-defined.

\section{Example}

To illustrate this approach, we explicitly consider the up transitions from state $\{00\}$ towards all other states following the three step process. (i) The design constraint Eq. (4) determines that the second hysteron will switch, so that $U^{c}=u_{2}^{+}$ and $S^{\prime}=\{01\}$. (ii) For $S^{\prime}$ to be stable at $U^{c}$, we require

$$
\begin{gathered}
U^{c}=u_{2}^{+}<u_{1}^{+}-c_{12}, \\
U^{c}=u_{2}^{+}>u_{2}^{-},
\end{gathered}
$$

where we note that the second condition is trivially satisfied due to the design constraint Eq. (6). Hence, we can conclude the following necessary and sufficient condition:

$$
\{00\} \rightarrow\{01\}: u_{2}^{+}<u_{1}^{+}-c_{12} .
$$

We note that for noninteracting hysterons, where $c_{12}=0$, this condition is trivially satisfied due to design condition Eq. (4). Moreover, this constraint can only be violated for positive $c_{12}>u_{2}^{+}-u_{1}^{+}$. More generally, "vertical" and "horizontal" avalanches require positive and negative coupling coefficients.

We now iterate this process, by considering the case when the transition state $\{01\}$ is not stable, i.e., when Eq. (9) is not satisfied. We then obtain a new transition state $S^{\prime}=\{11\}$, and we check the stability of $S^{\prime}$ :

$$
\begin{aligned}
& u_{2}^{+}>u_{1}^{-}-c_{12}, \\
& u_{2}^{+}>u_{2}^{-}-c_{21} .
\end{aligned}
$$

We note that Eq. (10) is automatically satisfied because of the design constraint Eq. (5) and the fact that Eq. (9) is not satisfied. Inequality (10) is thus redundant and can be removed from our considerations-such dependencies between 
inequalities on the design parameters frequently occur. Hence, the necessary conditions for an avalanche from $\{00\}$ to $\{11\}$ are

$$
\begin{aligned}
\{00\} & \rightarrow\{11\}: \\
u_{2}^{+} & >u_{1}^{+}-c_{12}, \\
u_{2}^{+} & >u_{2}^{-}-c_{21} .
\end{aligned}
$$

We now consider whether these equations are sufficient. For an avalanche, we do not keep track of the intermediate transition states and only monitor the initial and final state. In principle, the $\{00\} \rightarrow\{11\}$ avalanche might also have proceeded differently, e.g., via the $\{10\}$ state; in this specific case, the design constraint Eq. (4) blocks this possibility. For larger systems, finding sufficient and necessary conditions for avalanches becomes much more involved. However, in this specific case, Eqs. (12) and (13) are both sufficient and necessary conditions for the $\{00\} \rightarrow\{11\}$ avalanche.

We repeat this procedure again in case that state $\{11\}$ is unstable at $U=u_{2}^{+}$, which happens when Eq. (12) is satisfied, and Eq. (13) is violated, and which leads to a new transition state $\{10\}$. Checking the stability of this state yields

$$
\begin{gathered}
u_{2}^{+}<u_{2}^{+}-c_{21}, \\
u_{2}^{+}>u_{1}^{-} .
\end{gathered}
$$

We note that $c_{21}$ has to be negative to violate Eq. (13), which implies that Eq. (14) is satisfied and can be removed from our consideration. Hence, the conditions for the pseudoavalanche of length three $\{00\} \rightarrow\{10\}$ of the t-graph shown in Fig. 4(j) are

$$
\begin{aligned}
\{00\} & \rightarrow\{10\}: \\
u_{2}^{+} & >u_{1}^{+}-c_{12}, \\
u_{2}^{+} & <u_{2}^{-}-c_{21}, \\
u_{2}^{+} & >u_{1}^{-} .
\end{aligned}
$$

Finally, we note that violating the last equality Eq. (18) yields a cycle $\{00\} \rightarrow\{00\}$, which yields the t-graph to be ill-defined; this indeed can happen (a concrete realization would be $u_{1}^{+}=0.5, u_{1}^{-}=0.8, u_{2}^{+}=1, u_{2}^{-}=0.7, c_{12}=$ $\left.0.1, c_{21}=-0.4\right)$. We summarize these findings in a decision three [Fig. 5(b)], where we stress that for other states and for more hysterons, the situation generally is much more complex, featuring multiple conditions and outcomes per branch point, and cases where different branches yield the same transition.

After collecting all inequalities for all possible $n=2$ transitions, and removing redundant inequalities, we obtain necessary and sufficient conditions for each of the 11 possible t-graphs, as well as precise conditions for the occurrence of ill-defined graphs. We can express these by combining nine inequalities $x_{i}$, and we have checked these conditions numerically. We note that these inequalities $x_{i}$ are not independent - for example, $x_{1} \wedge x_{8} \Rightarrow x_{7}$ - and we have checked that only 78 different combinations of $\left\{x_{1}, \ldots, x_{9}\right\}$ arise.

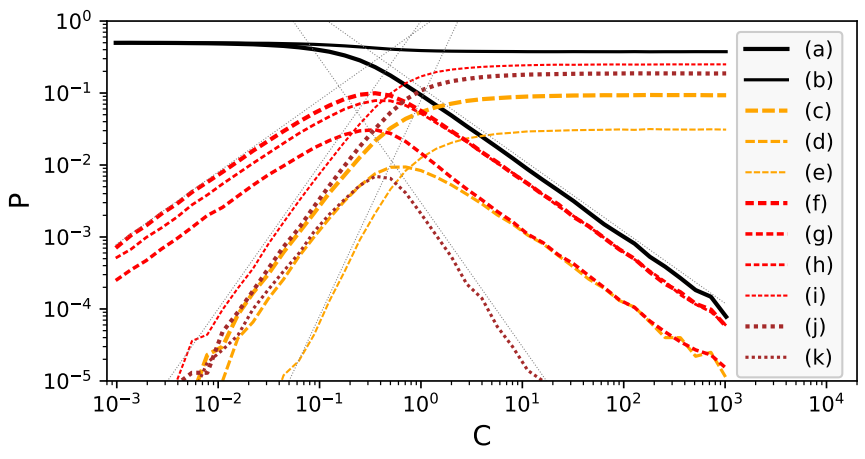

FIG. 6. Probability of each t-graph shown in Fig. 4 as function of magnitude of the interactions $C$; fractions are for well-defined cases only (the fraction of ill-defined cases grows from $0 \%$ at small $C$ to $6 \%$ at large $C$ ). Thin gray lines indicate integer powerlaw scalings.

The rather impenetrable nature of these sets of inequalities reflect the intrinsic complexity of the relation between design parameters and t-graphs. Nevertheless, we stress here that obtaining these inequalities is straightforward and could be automated. Solving these inequalities, i.e., finding design parameters for a given t-graph (potentially with additional constraints such as weak interactions, or with a number of interactions constants zero) can straightforwardly be implemented using linear programming. Finally, as we will see in the next section, these inequalities give crucial insight into the statistical properties of t-graphs.

\section{Statistics}

We have sampled the probability for each t-graph to occur as a function of $C$ (ensemble size $10^{8}$ ), for the "mixed interaction" case where $\left|c_{i j}\right| \leqslant C$ [Fig. 2(1)]. While the interactions constants $c_{i j}$ are flatly sampled, only restricted by simple independent constraints such as $\left|c_{i j}\right|<C$, the bare switching fields have to satisfy two sets of constraints: First, we require $u_{i}^{-}<u_{i}^{+}$so that independent hysterons are well-defined, and second, we require the ordering of the upper switching fields $\left(u_{1}^{+}>u_{2}^{+}>\ldots\right)$ which limits the number of t-graphs by suppressing trivial permutations of the hysterons. To numerically sample the switching fields that satisfy these constraints, we use an algorithm that guarantees that, for $c_{i j} \equiv 0$, all different orderings of the lower switching fields, and thus all Preisach t-graphs, occur with equal probability.

As shown in Fig. 6, our data shows that all t-graphs can be realized for arbitrary weak interactions. This can be understood from the invariance of the t-graph topology under shifts of the switching fields and multiplications of all parameters: The t-graph for $\bar{c}_{i j}=\lambda c_{i j}, \bar{u}_{i}^{ \pm}=\lambda\left(u_{i}^{ \pm}+1 / \lambda\right)$ maintains its topology for arbitrarily small interaction constants $(\lambda \rightarrow 0)$. Hence, for any set of parameters, we can find other parameters with arbitrarily small $c_{i j}$ such that the t-graph topology is maintained. Crucially, this shows that weak interactions can break the Preisach phenomenology when the switching fields are close to each other.

Moreover, we find that these probabilities grow and decay as powerlaws $\sim C_{i}^{n}$ for small and large $C$, with integer exponents $n_{i}$. This is because some of the design inequalities are "critical" and require the fine tuning of parameters when 
$c_{i j}$ is large or small. As an example, consider condition $x_{1}$ : $u_{2}^{+}-u_{1}^{+}>-c_{12}$. As the design constraint Eq. (4) stipulates that $u_{2}^{+}<u_{1}^{+}, x_{1}$ can only be satisfied when $c_{12}$ is positive, and when $c_{12} \downarrow 0$ requires the difference between $u_{1}^{+}$and $u_{2}^{+}$to become vanishingly small, which statistically happens with probability $\mathcal{O}(|c|)$ - hence, $x_{1}$ is a critical condition for small $|c|$. Similarly, conditions $x_{5}, \neg x_{6}$ and $\neg x_{8}$ also are satisfied with probability $\mathcal{O}(|c|)$. Moreover, some combinations of condition may only occur with probability $\mathcal{O}(|c|)$. When $m$ independent critical conditions occur, t-graphs can only arise with probability $\mathcal{O}\left(|c|^{m}\right)$. Similarly, when $|c|$ becomes very large, some (combinations) of the inequalities $x_{i}$ can only be satisfied when the coupling constants are of order one, which happens with probability $\mathcal{O}\left(|c|^{-1}\right)$. Together, these considerations explain the power law behavior seen in Fig. 6, with the number of independent critical conditions controlling $n_{i}$.

We finally note that while the details of the scaling of each t-graph may be intricate, the data in Fig. 6 suggests that understanding both the small $C$ Preisach limit, as well as the large $C$ limit is sufficient to capture more of the trends. For large $C$, the interaction coefficients, $c_{12}$ and $c_{21}$ completely dominate all state switching fields $U_{i}^{ \pm}$, with the bare switching fields acting as perturbations; while it's physical interpretation is not immediately clear, studying this limit in tandem with the small $C$ limit may provide insight into the statistical properties of (groups of) t-graphs.

\section{THREE INTERACTING HYSTERONS}

The number and qualitative diversity of t-graphs mushrooms with $n$. We determined t-graphs for $10^{8}$ systems of $n=3$ hysterons for $C=1$. We stress that in absence of interactions, there are only $n !=6$ distinct t-graphs [20,21]. Strikingly, in the presence of interactions we obtain more than 15,000 distinct t-graphs. To categorize the topology of these t-graphs, we focus on three characterizations: the nature of the transitions, the nature of pairs of transitions, and global topological measures of the t-graph. In particular, individual transitions can feature avalanches, where more than one hysteron changes state simultaneously, and pairs of transitions can be scrambled, meaning that the switching order of hysterons becomes state dependent. These "local" features can lead to global t-graph topologies absent in the Preisach model, including the break-down of loop-Return Point Memory, subharmonic cycles, and t-graphs that are multigraphs. Together, these new features open up a large space of essentially unexplored behavior.

\section{A. Main features of t-graphs of coupled hysterons}

To illustrate the main new features of t-graphs due to interactions, we present nine examples of t-graphs that together illustrate the essential features [Figs. 7(a)-(i)] (see Supplemental Material [35]).

(i) Scrambling. In the Preisach model, the switching order is independent of state. We define two nonavalanche transitions as scrambled when they are inconsistent with such a state-independent ordering. For example, consider the pair of transitions $\{011\} \rightarrow\{001\}$ and $\{111\} \rightarrow\{110\}$ in the t-graph
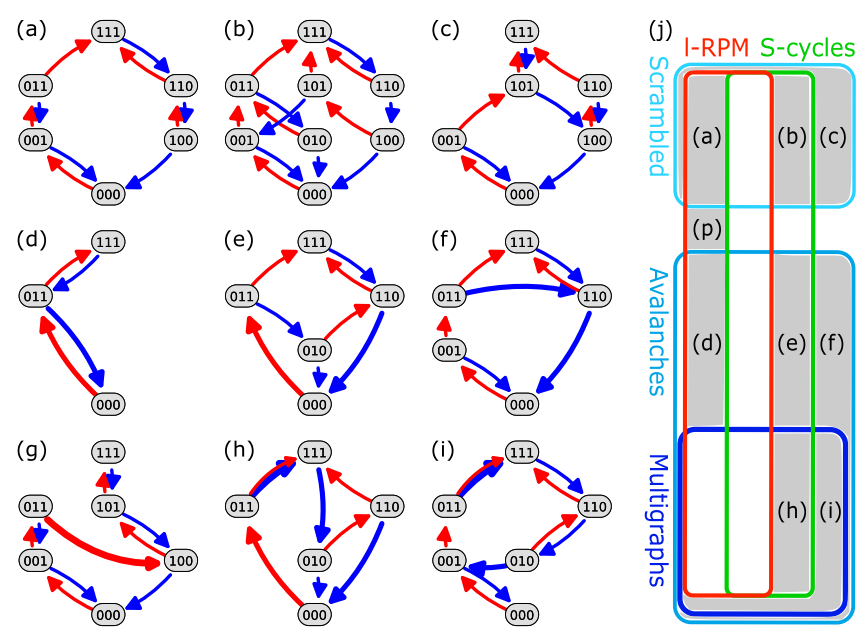

FIG. 7. (a-c) Examples of scrambled t-graphs, featuring either l-RPM (a), a subharmonic-cycle (b), or neither (c). (d-f) Examples of t-graphs with avalanches (thick arrows), featuring either 1-RPM (d), a subharmonic-cycle (e), or neither (f). (g) t-graph featuring a dissonant avalanche. (h, i) Multigraphs with (h) and without (i) a subharmonic cycle. (j) Venn-diagram for properties of $n=3 \mathrm{t}$-graphs; letters refer to the examples in earlier panels, while Preisach t-graphs are indicated by “(p)."

in Fig. 7(a). The presence of the first transition implies that $U_{2}^{-}(011)>U_{3}^{-}(011)$, and the second implies that $U_{2}^{-}(111)<$ $U_{3}^{-}(111)$. Such a pair of inequalities of the form $U_{i}^{ \pm}\left(S_{1}\right)>$ $U_{j}^{ \pm}\left(S_{1}\right)$ and $U_{i}^{ \pm}\left(S_{2}\right)<U_{j}^{ \pm}\left(S_{2}\right)$ can only occur due to hysteron interactions, and we label the pair of corresponding transitions as scrambled. We note that when avalanches are present, their intermediate steps are "hidden," hindering to establish whether such a transitions are part of a scrambled pair of transitions-for the notion of scrambling, we therefore focus on pairs of direct (i.e., nonavalanche) transitions.

(ii) Avalanches. Without interactions, each transition corresponds to a single hysteron switching its phase, but in the presence of interactions many t-graphs feature avalanches where more than one hysteron changes phase simultaneously [Figs. 7(d)-(f)]. We note that ferromagnetic interactions promote "vertical" avalanches, where multiple hysterons collectively switch up or down. The magnetization $m(S):=\Sigma_{i} s_{i}$ then increases or decreases by more than one. In contrast, antiferromagnetic interactions promote "horizontal" avalanches, where the magnetization remains constant or changes at most by one. Mixed interactions in addition can lead to more complex avalanches, such as the "pseudoavalanches" shown in Figs. 4(j) and 4(k).

(iii) Dissonance. So far, up and down transitions, initiated by an increase or decrease of the global driving $U$, lead to the increase (respectively, decrease) of the magnetization $m$. Remarkably, mixed ferro/antiferromagnetic interactions allow for dissonant avalanches, where an up (down) avalanche leads to a decrease (increase) of the magnetization [Fig. 7(g)].

These three features significantly extend the space of possible t-graphs in comparison to those found in the Preisach model. Scrambling breaks the notion of a unique switching ordering, avalanches break the notion of nearby states, and dissonance blurs the connection between (in)decrease of the 
driving field, and in(decrease) of the number of hysterons in state "1." Collectively, these features lead to a range of new global behaviors of the t-graphs:

(iv) Multigraphs. The presence of avalanches and dissonant transitions leads to cases where two states are connected both by an up and down transition-e.g., a pair of "horizontal" avalanches that both preserve the magnetization, or an ordinary transition paired with a dissonant avalanche. The t-graphs then become directed multigraphs [Figs. 7(h) and 7(i)]. We stress here that it is essential for the algorithm that constructs t-graphs to allow for such multigraphs, which are surprisingly common for intermediate coupling coefficients.

(v) Breakdown of loop-Return point memory. Return point memory (RPM) occurs in a range of physical systems and in particular has been widely studied for the Preisach model [17,20,21]. Loosely speaking, a system exhibits RPM when it revisits a previous state when the driving revisits a previous minimum or maximum of the driving. A t-graph satisfies RPM when one cannot escape a subloop without the driving passing through some previously established extremal values [20,21]. While the presence of strict RPM may depend on the precise values of the switching fields [13], a recent definition of socalled loop-RPM (l-RPM) focusses on the topology of the t-graph [20,21]. Essentially, 1-RPM requires that each loop, given by a pair of "top" and "bottom" states connected by two sequences of purely up and a down transitions, is "absorbing": this requires that any orbit starting from a state in this loop escapes the loop by going to either the top or bottom state, and not differently (see Supplemental Material [35] for the precise definition). This definition is clearly analogous of that of RPM, and we note here that although RPM implies 1-RPM, the converse is not necessarily true [13]. The t-graphs of the Preisach model all satisfy both properties, and ferromagnetic interactions have long been known to preserve RPM due to the so-called no-passing property [17,20,21].

Figures 4(a), 4(d) and 4(g) satisfy l-RPM: (antiferromagnetic) interactions frequently break l-RPM. For example, in Fig. 7(c) the transitions $\{100\} \rightarrow\{110\} \rightarrow\{111\}$ escape the subloop between nodes $\{000\}$ and $\{101\}$ (in the SI we describe for all other t-graphs the precise transitions that break l-RPM). We finally note that the presence of either scrambling or avalanches is a necessary, but not sufficient condition to break 1-RPM.

(vi) Subharmonic cycles. Scrambling may also lead to subharmonic cycles (S-cycles), where under cyclic driving the system revisits earlier states only after more than one driving cycle. Similar to the discussion on 1-RPM, we require here a definition in terms of the t-graphs topology, without regard to the precise switching values. Hence, we say the graph has an S-cycle if there are sequences of up and down transitions where one returns to the beginning state under more than one up/down subsequence. Figures 7(b), 7(e) and 7(h) show t-graphs with such S-cycles; for example, the tgraph of Fig. 7(b) contains an S-cycle of period two: $\{001\} \uparrow$ $\{011\} \uparrow\{111\} \downarrow\{110\} \downarrow\{100\} \uparrow\{101\} \downarrow\{001\} \ldots$ where $\uparrow$ and $\downarrow$ denote up and down transitions.

(vii) Absence of l-RPM and S-cycles. While we observe that 1-RPM and the presence of S-cycles are mutually exclusive for $n=3$, we stress here that it is also possible to break 1-RPM without having a S-cycle, as shown in Figs. 7(c), 7(f) and 7(i).
The presence of scrambling or avalanches is necessary, but clearly not sufficient to break 1-RPM or obtain S-cycles. Beyond that, we find that, at least for $n=3$, the "local" measures-scrambling, avalanches, multigraphs-can occur concurrently with the "global" measures, l-RPM and the presence of S-cycles, except that multigraphs can never satisfy l-RPM [Fig. 7(j)]. This can easily be understood by noting that a multiedge in a given loop implies that an up-boundary contains a down transition, or a down-boundary contains an up-transition, which allows to establish an orbit that violates 1-RPM.

In Table II we present examples of switching fields and coupling coefficients that produce t-graphs with the same topologies as shown in Fig. 7. These parameter values have been selected after some manual optimization steps, setting some small interactions to zero and rounding of all values to at most two significant digits. While these parameters are not optimal in any well-defined sense, they may serve as specific starting points for further studies, as well as to guide the reader in the construction of t-graphs by providing specific examples. Moreover, we have numerically checked that for these parameters, small changes of $\mathcal{O}\left(10^{-3}\right)$ do not change the topology of the t-graph, thus demonstrating that even rare graphs are robust.

In summary, these examples illustrate how hysteron interactions generate a host of new features of the t-graphs. In particular, scrambling breaks the state independent ordering of transitions seen in Preisach t-graphs, avalanches and dissonance enlarge the types of transitions between states, and together these can yield multigraphs, breakdown of 1-RPM and subharmonic cycles.

\section{B. Statistics}

For $C=1$, more that $62 \%$ of distinct t-graphs break 1RPM. However, not all of these t-graphs are statistically equally likely. To probe the statistical properties, we have sampled the probability of 1-RPM, avalanches, scrambling, $\mathrm{S}$-cycles, multigraphs and ill-defined cases as function of $C$ for an ensemble size $10^{5}$ (Fig. 8). Strikingly, the majority of random parameters yield t-graphs that satisfy 1-RPM (minimum fraction $\sim 0.93$ for $C \approx 0.4$ ). Hence, while a fraction of all t-graphs dominates the statistics, interactions produce a wide variety of t-graphs.

The probabilities of each class of t-graphs vary similarly to the probability of individual $\mathrm{t}$-graphs, with integer powerlaws $\sim C^{n}$, and also point to well defined behavior in the $C \rightarrow \infty$ limit. Most interesting behavior occurs for $C$ between 0.1 and 1 , where the probability of scrambled transitions, $\mathrm{S}$-cycles and multigraphs peaks. For large $C$ the number of ill-defined t-graphs plateaus at $57 \%$, and explorations for larger $n$ indicate even larger percentages. This suggests that additional rules that avoid ill-defined transitions and/or loops are necessary to study the behavior of larger systems with strong interactions. Finally, for the remaining $43 \%$ of parameters that yield welldefined t-graphs, most lead to t-graphs with avalanches and which satisfy l-RPM.

We have further explored differences between purely ferromagnetic, purely antiferromagnetic, and mixed interactions. First, we demonstrate that the statistical weight of 
TABLE II. Examples of switching fields and coupling coefficients that produce the t-graphs shown in Fig. 7.

\begin{tabular}{llllllllllll}
\hline \hline Panel & $u_{1}^{+}$ & $u_{2}^{+}$ & $u_{3}^{+}$ & $u_{1}^{-}$ & $u_{1}^{-}$ & $u_{1}^{-}$ & $c_{12}$ & $c_{13}$ & $c_{21}$ & $c_{23}$ & $c_{31}$ \\
\hline (a) & 0.9 & 0.6 & 0.5 & 0.2 & 0.4 & 0.1 & 0 & 0 & 0 & 0 & -0.2 \\
(b) & 1 & 0.8 & 0.6 & 0.55 & 0 & 0.4 & -0.1 & -0.4 & -0.75 & -0.45 & -0.4 \\
(c) & 1 & 0.9 & 0.8 & 0.2 & 0.8 & 0 & 0 & 0 & 0 & -0.15 & -0.3 \\
(d) & 1 & 0.7 & 0.5 & 0.3 & 0.6 & 0.4 & 0.0 & -0.15 & 0 & 0.25 & 0 \\
(e) & 1 & 0.9 & 0.8 & 0.3 & 0.2 & 0.5 & 0.25 & -0.2 & 0 & 0.2 & 0 \\
(f) & 0.9 & 0.7 & 0.6 & 0 & 0.1 & 0.3 & 0.1 & -0.3 & 0.3 & -0.25 & 0 \\
(g) & 0.9 & 0.7 & 0.5 & 0.1 & 0.4 & 0.35 & 0.3 & -0.4 & -0.7 & 0 & -0.7 \\
(h) & 1 & 0.95 & 0.9 & 0.55 & 0.6 & 0.7 & 0.23 & -0.3 & 0.1 & 0.4 & 0.3 \\
(i) & 0.75 & 0.6 & 0.3 & 0.35 & 0.39 & 0.28 & -0.05 & -0.30 & 0 & -0.1 & -0.1 \\
\hline \hline
\end{tabular}

individual t-graphs is broadly distributed, by studying $10^{8}$ t-graphs realized for interactions strengths $C=0.3$ and $C=$ 1 and for mixed $\left(-C<c_{i j}<C\right)$, ferromagnetic $\left(0<c_{i j}<C\right)$, and purely antiferromagnetic $\left(-C<c_{i j}<0\right)$ interactions. By ordering each t-graph by its probability (from high to low), we observe that the probabilities for a given t-graph span many decades, with the majority of t-graphs spanning a small fraction of parameter space [Fig. 9(a)].

Second, and consistent with the profusion of rare t-graphs, we find that the number of distinct t-graphs as function of the number of samples grows slowly [Fig. 9(b)]. In particular, while for $10^{8}$ realizations the number of t-graphs for purely ferromagnetic and antiferromagnetic appears has (nearly) saturated around 198 and $\mathcal{O}(4050)$, the number of t-graphs for mixed interactions is still growing [Fig. 9(b)]. Hence, exhaustive sampling, or smarter techniques to map out the space of t-graphs can be expected to yield even more, rare t-graphs.

Third, we observe purely ferromagnetic interactions do not generate S-cycles, multigraphs or dissonant avalanches and only produce t-graphs that satisfy l-RPM (possibly with "vertical" avalanches). This is completely consistent with earlier observations that ferromagnetic interactions preserve the no-passing property, severely restricting the t-graphs and pathways [16-21]. In contrast, purely antiferromagnetic can break l-RPM, generate S-cycles, and yield multigraphs, but

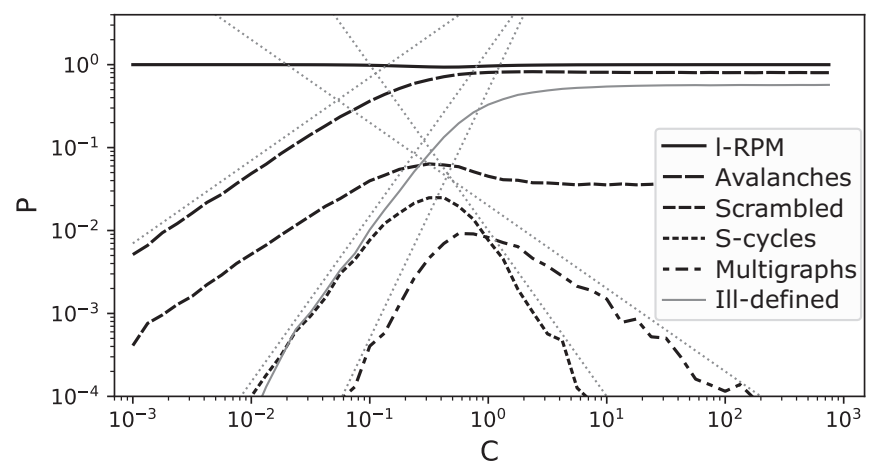

FIG. 8. Probabilities of $n=3 \mathrm{t}$-graph types, where $c_{i j} \in[-C, C]$. These probabilities grow and decay as integer power-laws (dashed lines). While scrambling, S-cycles, and multigraphs most likely arise for intermediate interactions, the fraction of t-graphs featuring avalanches increases with $C$ and plateaus at $80 \%$. The fraction of parameters that yield ill-defined t-graphs also increases with $C$ and plateaus at $57 \%$; fractions are for well-defined cases only. cannot create dissonant avalanches. Together, this shows that while antiferromagnetic interactions are essential to obtain exotic behavior, mixed interactions produce the largest variety of t-graphs.

Together, interacting hysterons lead to a large space of essentially unexplored t-graphs. Three features stand out. First, while statistically, 1-RPM is the most likely global behavior, even for strong coupling, a large number of t-graphs with qualitatively different features can be found. Second, scrambling breaks and avalanches can break the unique ordering in the switching sequences, and this is a necessary, although not sufficient property to break 1-RPM, and as such can be seen as the first step in a hierarchy of increasing complexity. Third, dissonant avalanches break the link between up and down transitions in increase and decrease of the magnetization, and open up the possibility of multigraphs, which never satisfy l-RPM and lead to even more strongly nonclassical behavior.

\section{DESIGNER PATHWAYS}

We suggest that the complex pathways of interacting hysterons naturally can be described in the language of computing. In particular, the directed graphs that encode sequential computations in finite state machines [34] are strongly reminiscent of t-graphs, where the labels of each edge ("up transition at $U=0.5$ ") play the role of the input to the "hysteron machine."

As a first example of such a hysteron machine we exploit dissonant avalanches to realize t-graphs that contain all eight states in a single pathway of up (or down) transitions. In our dataset, 740 realizations representing 51 distinct t-graphs contain such pathways. We select an example where both the up and down pathways between $\mathbf{0}$ and $\mathbf{1}$ follow the ordered binary numbers 000-111, and which acts as an analog-digital converter (ADC) [Fig. 10(a)]. The design inequalities specify a linear programming problem [25], and a judicious choice of parameters (see Table III) allows to tune the critical switching fields of the seven up and seven down transitions exactly to values $0.1,0.2, \ldots, 0.7$, respectively, $0.65,0.55, \ldots, 0.05$, making all states easily addressable and all transitions between states hysteretic (as required for ADCs). We have in addition verified that for the this design the t-graph's topology is stable to random perturbations of the design parameters of at least magnitude $10^{-3}$. As a second example, we explore the breakdown of 1-RPM and select an "accumulator" t-graph that contains the pathway $\{001\} \uparrow\{011\} \downarrow\{010\} \uparrow$ 

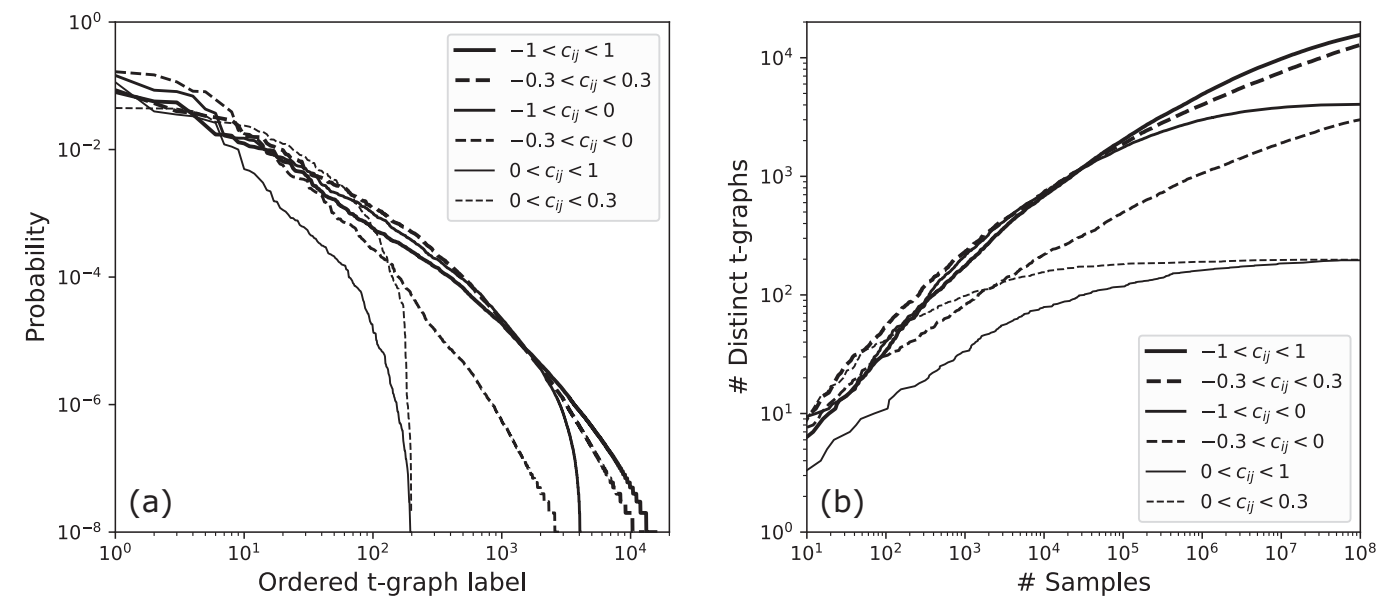

FIG. 9. Statistical measures of $n=3$ t-graphs, for two different interactions strengths and for mixed, ferromagnetic, and purely antiferromagnetic interactions. (a) Individual t-graphs occur with probabilities that can span at least four orders of magnitude. (b) As a function of the number of realizations, the number of t-graphs grows slowly, and for mixed interactions has not saturated for $10^{8}$ samples.

$\{110\} \uparrow\{100\}$ [Figs. 4(b) and 4(c)]. Hence, under cyclic driving, a system described by such a t-graphs "counts to two." This behavior has recently been observed by us in experiments, and may be related to transient memory $[13,26]$.
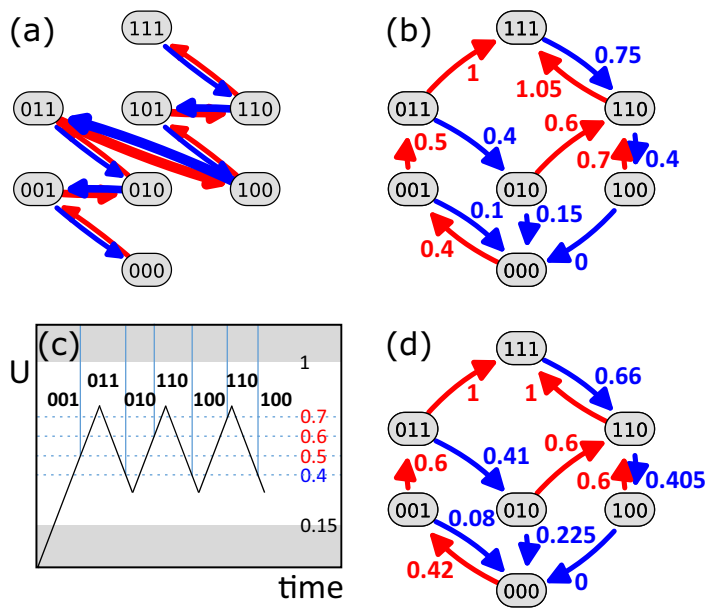

FIG. 10. (a) T-graph that sequentially accesses states by sweeping $U$ (we have changed the curvature of the arrows for visibility). (b) Accumulator t-graph with switching fields as indicated [Table III(b)]. (c) Response for the t-graphs shown in panel (b), demonstrating that for $0.7<U_{M}<1$, states $\{110\}$ and $\{100\}$ are only reached after two driving cycles. Dashed lines indicate critical switching fields, and gray regions indicate potential transitions to the extremal states. (d) Switching fields corresponding to an alternative design for the accumulator [Table III(d)].
This example is particularly rich, as even for a given topology, different response can be encoded depending on the precise values and orderings of the switching field. To demonstrate this, we first choose parameters such that the switching fields of the two down transitions $\{011\} \downarrow\{010\}$ and $\{110\} \downarrow\{100\}$ are equal to 0.4 , while the switching fields for $\{001\} \uparrow\{011\},\{010\} \uparrow\{110\}$, and $\{100\} \uparrow\{110\}$ are equal to $0.5,0.6$, and 0.7 , respectively [Table III(b)]. We have verified that the t-graph's topology is stable to random perturbations of the design parameters of magnitude $10^{-3}$.

The response of this system when $U$ is cycled between $u_{m}>0.15$ and $u_{M}<1$ evidences different accumulator/counting behavior. For $0.7<U_{M}<1$, the system reaches state $\{011\}$ at the first peak, and state $\{110\}$ at subsequent peaks: this pathway distinguishes between one or more cyclical drivings [Fig. 10(c)]. Moreover, for $0.6<U_{M}<0.7$, the first cycle reaches $\{011\}$, the second $\{110\}$ and subsequent cycles remain stuck at $\{100\}$ ("counting to three"); for $0.5<U_{M}<0.6$, the first cycle reaches $\{011\}$ and subsequent cycles remain stuck at $\{010\}$. Hence, a collection of three hysterons with appropriate interactions and switching fields can accumulate and count to two or three, depending on driving amplitude.

The parameters in our model offer freedom in the choice of the critical switching fields, although there are some constraints. For example, requiring that all three relevant up transitions in the accumulator t-graph $(\{001\} \uparrow\{011\},\{010\} \uparrow$ $\{110\},\{100\} \uparrow\{110\})$ are equal, necessitates the two down transitions $(\{011\} \downarrow\{010\},\{110\} \downarrow\{100\})$ to be unequal. To see this, we notice that in terms of the design parameters, the three up transitions are at $u_{2}^{+}-c_{23}, u_{1}^{+}-c_{12}$ and $u_{2}^{+}-c_{21}$,

TABLE III. Examples of switching fields and coupling coefficients that produce the t-graphs shown in Fig. 10.

\begin{tabular}{llllllllllll}
\hline \hline Panel & $u_{1}^{+}$ & $u_{2}^{+}$ & $u_{3}^{+}$ & $u_{1}^{-}$ & $u_{1}^{-}$ & $u_{1}^{-}$ & $c_{12}$ & $c_{13}$ & $c_{21}$ & $c_{23}$ & $c_{31}$ \\
\hline (a) & 0.8 & 0.4 & 0.1 & 0.35 & 0.15 & 0.05 & 0 & 0 & 0 & 0 & -0.2 \\
(b) & 0.7 & 0.45 & 0.4 & 0 & 0.15 & 0.1 & 0.1 & -0.4 & -0.25 & -0.05 & -0.35 \\
(d) & 0.7 & 0.45 & 0.42 & 0 & 0.255 & 0.08 & 0.1 & -0.4 & -0.15 & -0.3 \\
\hline \hline
\end{tabular}


respectively, so that when all are equal, $c_{21}=c_{23}$. Similarly, for the down transitions to have equal switching fields, we require $u_{3}^{-}-c_{32}=u_{2}^{-}-c_{21}$. Finally, for the down transition from state $\{011\}$ land on state $\{010\}$ and not on $\{001\}$, we require the design inequality $u_{3}^{-}-c_{32}>u_{2}^{-}-c_{23}$. When the pair of down transitions are equal, this latter inequality can be rewritten as $u_{2}^{-}-c_{21}>u_{2}^{-}-c_{23} \Rightarrow c_{21}<c_{23}$, which is in disagreement with the requirement that all three up transitions are at the same switching field.

Nothwithstanding this constraint, it is easy to find design parameters so that the three up-transitions are equal [Table III(d)], yielding the critical switching fields shown in Fig. 10(d). As all the relevant up switching fields are equal, the only counting behavior that is left is "counting to two." This example demonstrates that even for a given t-graph topology, qualitatively distinct responses and finite state machines may be encoded.

We suggest that a wide variety of more complex functions may be achievable in t-graphs that encode different topologies, such as (longer) S-cycles, multiple S-cycles, etc. Paired with the design options that e.g., tune all relevant up transition fields to the same value or specifically ordered values, the design space for complex hysteron "machines" is very large.

\section{DISCUSSION}

This work highlights that even small collections of weakly interacting hysterons exhibit a staggering multitude and variety of pathways and t-graphs, and suggests that hysterons with appropriate thresholds and interactions can act as information processing devices. We highlight a number of key questions. First, the types of t-graphs and underlying computations that can be realized by interacting hysterons is unknown, with interesting subquestions arising for interactions that are purely ferromagnetic, purely antiferromagnetic, reciprocal $\left(c_{i j}=c_{j i}[25]\right)$, or sparse. Second, in exploratory studies we have found that the fraction of random parameters that yield ill-defined t-graphs increases with $n$ and $C$ and asymptotes to one. This suggests that strongly coupled systems cannot trivially be described by hysterons, and more advanced models, that avoid ambiguities due to multiple unstable hysterons or self-loops, are called for. Third, metamaterials might yield physical realizations, with serially coupled mechanical hysterons naturally implementing antiferromagnetic interactions [27,31,33]. Finally, viscoelastic effects could be leveraged to obtain rate-dependent pathways and t-graphs and self-learning systems [36-40]. Together, progress on these questions will realize targeted pathways and information processing in designer materials.

\section{ACKNOWLEDGMENTS}

We acknowledge insightful discussions with $\mathrm{H}$. Bense, $\mathrm{K}$. Bertoldi, N. Keim, G. Korovin, Y. Lahini, C. Lindeman, M. Mungan, S. Nagel, J. Paulsen, and M. Zanaty.
[1] E. M. Kramer and T. A. Witten, Phys. Rev. Lett. 78, 1303 (1997).

[2] E. Cerda, S. Chaieb, F. Melo, and L. Mahadevan, Nature (London) 401, 46 (1999).

[3] K. Matan, R. B. Williams, T. A. Witten, and S. R. Nagel, Phys. Rev. Lett. 88, 076101 (2002).

[4] T. A. Witten, Rev. Mod. Phys. 79, 643 (2007).

[5] H. Aharoni and E. Sharon, Nat. Mater. 9, 993 (2010).

[6] N. Oppenheimer and T. A. Witten, Phys. Rev. E 92, 052401 (2015).

[7] Y. Lahini, O. Gottesman, A. Amir, and S. M. Rubinstein, Phys. Rev. Lett. 118, 085501 (2017).

[8] K. A. Murphy, J. W. Kruppe, and H. M. Jaeger, Phys. Rev. Lett. 124, 168002 (2020).

[9] R. Candelier and O. Dauchot, Phys. Rev. Lett. 103, 128001 (2009).

[10] S. Slotterback, M. Mailman, K. Ronaszegi, M. van Hecke, M. Girvan, and W. Losert, Phys. Rev. E 85, 021309 (2012).

[11] N. C. Keim and S. R. Nagel, Phys. Rev. Lett. 107, 010603 (2011).

[12] D. Bonn, M. M. Denn, L. Berthier, T. Divoux, and S. Manneville, Rev. Mod. Phys. 89, 035005 (2017).

[13] H. Bense and M. van Hecke, arXiv:2106.14441 (2021).

[14] J. P. Sethna, K. A. Dahmen, and C. R. Myers, Nature (London) 410, 242 (2001).

[15] F. Preisach, Z. Phys. 94, 277 (1935).

[16] J. A. Barker, D. E. Schreiber, B. G. Huth, and D. H. Everett, Proc. R. Soc. London A 386, 251 (1983).

[17] A. A. Middleton, Phys. Rev. Lett. 68, 670 (1992).
[18] J. P. Sethna, K. Dahmen, S. Kartha, J. A. Krumhansl, B. W. Roberts, and J. D. Shore, Phys. Rev. Lett. 70, 3347 (1993).

[19] N. C. Keim, J. D. Paulsen, Z. Zeravcic, S. Sastry, and S. R. Nagel, Rev. Mod. Phys. 91, 035002 (2019).

[20] M. Mungan and M. M. Terzi, Ann. H. Poincare 20, 2819 (2019).

[21] M. M. Terzi and M. Mungan, Phys. Rev. E 102, 012122 (2020).

[22] M. Mungan, S. Sastry, K. Dahmen, and I. Regev, Phys. Rev. Lett. 123, 178002 (2019).

[23] I. Regev, I. Attia, K. Dahmen, S. Sastry, and M. Mungan, Phys. Rev. E 103, 062614 (2021).

[24] J. D. Paulsen and N. C. Keim, Proc. R. Soc. London A 475, 20180874 (2019).

[25] N. C. Keim and J. D. Paulsen, Sci. Adv. 7, eabg7685 (2021)

[26] C. W. Lindeman and S. R. Nagel, Sci. Adv. 7, eabg7133 (2021).

[27] T. Jules, A. Reid, K. E. Daniels, M. Mungan, and F. Lechenault, arXiv:2106.08243 (2021).

[28] K. Bertoldi, V. Vitelli, J. Christensen, and M. van Hecke, Nat. Rev. Mater. 2, 17066 (2017).

[29] C. Coulais, A. Sabbadini, F. Vink, and M. van Hecke, Nature (London) 561, 512 (2018).

[30] M. Stern, V. Jayaram, and A. Murugan, Nat. Commun. 9, 4303 (2018).

[31] M. Zanaty and K. Bertoldi (private communication).

[32] D. Shohat, D. Hexner, and Y. Lahini, arXiv:2109.05212 (2021).

[33] J. Liu, G. Korovin, Z. Vermaire, H. Bense, and M. van Hecke (unpublished). 
[34] J. Sakavorith, Elements of Automata Theory (Cambridge University Press, London, 2009).

[35] See Supplemental Material at http://link.aps.org/supplemental/ 10.1103/PhysRevE.104.054608 for the definition of 1-RPM and a detailed description of the properties of the t-graphs shown in Fig. 7.

[36] D. Dykstra, J. Busink, B. Ennis, and C. Coulais, J. Appl. Mech. 86, 111012 (2019).
[37] S. Janbaz, K. Narooei, T. van Manen, and A. A. Zadpoor, Sci. Adv. 6, eaba0616 (2020).

[38] A. Bossart, D. M. J. Dykstra, J. van der Laan, and C. Coulais, Proc. Natl. Acad. Sci. USA 118, e2018610118 (2021).

[39] N. Pashine, D. Hexner, A. J. Liu, and S. R. Nagel, Sci. Adv. 5, eaax4215 (2019).

[40] M. Stern, D. Hexner, J. W. Rocks, and A. J. Liu, Phys. Rev. X 11, 021045 (2021). 